\title{
基于注意力和多标签分类的图像实时语义分割
}

\author{
高翔，李春庚，安居白 \\ (大连海事大学信息科学技术学院 大连 116026) \\ (li_chungeng@dlmu.edu.cn)
}

\begin{abstract}
摘 要：针对现阶段很多实时语义分割算法分割精度低，尤其对边界像素分割模糊的问题，提出一种基于跨级注意 力机制和多标签分类的高精度实时语义分割算法. 首先基于 DeepLabv3 进行优化, 使其达到实时运算速度. 然后在 此网络基础上增加跨级注意力模块, 使深层特征为浅层特征提供像素级注意力, 以抑制浅层特征中不准确语义信息 的输出; 并在训练阶段引人多标签分类损失函数辅助监督训练. 在 Cityscapes 数据集和 CamVid 数据集上的实验结果 表明, 该算法的分割精度分别为 $68.1 \%$ 和 $74.1 \%$, 分割速度分别为 42 帧 $/ \mathrm{s}$ 和 89 帧/s, 在实时性与准确性之间达到较好 的平衡，能够优化边缘分割，在复杂场景分割中具有较好的鲁棒性.
\end{abstract}

关键词: 卷积神经网络; 实时语义分割; 多标签分类; 跨级注意力机制

中图法分类号: TP391.4 DOI: 10.3724/SP.J.1089.2021.18233

\section{Real-Time Image Semantic Segmentation Based on Attention Mechanism and Multi-Label Classification}

Gao Xiang, Li Chungeng ${ }^{*}$, and An Jubai

(College of Information Sciences and Technology, Dalian Maritime University, Dalian 116026)

\begin{abstract}
Improving the accuracy is the goal in real-time semantic segmentation, especially for fuzzy boundary pixel segmentation. We proposed a high-precision and real-time semantic segmentation algorithm based on cross-level attention mechanism and multi-label classification. The procedure started with an optimization of DeepLabv3 to achieve real-time segmentation speed. Then, a cross-level attention module was added, so that the high-level features provided pixel-level attention for the low-level features, so as to inhibit the output of inaccurate semantic information in the low-level features. In the training phase, the multi-label classification loss function was introduced to assist the supervised training. The experimental results on Cityscapes dataset and CamVid dataset show that the segmentation accuracy is $68.1 \%$ and $74.1 \%$ respectively, and the segmentation speed is 42 frames/s and 89 frames/s respectively. It achieves a good balance between segmentation speed and accuracy, can optimize edge segmentation, and has strong robustness in complex scene segmentation.
\end{abstract}

Key words: convolutional neural networks; real-time semantic segmentation; multi-label classification; cross-level attention mechanism

收稿日期: 2020-02-16; 修回日期: 2020-05-23. 基金项目: 国家自然科学基金(61471079). 高翔(1994一), 女, 硕士研究生, 主要 研究方向为深度学习图像语义分割; 李春庚(1969-), 男, 博士, 副教授, 硕士生导师, 论文通讯作者, 主要研究方向为数字图像处理、基 于视频的运动目标追踪; 安居白(1958-), 男, 博士, 教授, 博士生导师, 主要研究方向为模式识别、海上遥感图像分析. 
图像语义分割是计算机视觉的一项重要技术, 相比图像分类和目标检测，它是一种更细粒度的 像素级分类技术 ${ }^{[1]}$, 该技术在生产环境中具有实现 成本低、部署方便的优点, 因此在无人驾驶、机器 人视觉等领域 ${ }^{[2-3]}$ 常常被应用于可行驶区域的感知 系统，这些应用领域对快速交互或响应速度有很 高的要求.

文献[4]提出使用全卷积神经网络(fully convolutional networks, FCN)实现端到端的语义分割, 通过卷积和池化层对输人图像逐步下采样获得具 有强鲁棒性的特征，但也导致特征分辨率降低，对 目标边界的分割不够精细. 此后，为更加精确地恢 复高分辨率的特征, 文献 [5-6]使用编码器来获得 深层特征的语义信息, 使用解码器融合浅层和深 层特征，逐步恢复空间和细节信息. 此外，文献[7] 提出放弃编码器最后 2 次下采样操作, 使用空洞卷 积保持算法的整体感受野不变, 并在网络末端增 加全连接条件随机场进一步精细化网络的分割结 果. 为避免特征图分辨率变小、定位精度过低等问 题, DeepLabv2 $2^{[8]}$ 将空洞卷积与空间金字塔池化方 法结合，提出空洞空间金字塔池化(atrous spatial pyramid pooling, ASPP)模块整合多尺度特征、增大 感受野，进而提高分割精度. 基于以上 2 种方法， DeepLabv3 $3^{[9]}$ 进一步讨论了空洞卷积的并联和串联 方式对算法分割效果的影响，改进 ASPP 模块，进 而获取不同感受野信息，提高了分割不同尺度目 标的能力, 取得更好的语义分割效果. 文献[10]认 为，丰富的上下文信息可以增强网络的信息丰富 度与类别区分度，使得网络模型具有更好的语义 分割能力.

以上工作主要解决因网络下采样造成特征的 空间信息丢失问题，虽然提高了分割精度，但是分 割速度较慢，无法满足实时分割任务需求. 目前实 时语义分割算法大都以牺牲分割精度为前提达到实 时分割速度. 实时语义分割算法 $S e g N t^{[11]}$ 采用编码 器-解码器结构, 在编码过程中通过多次卷积与池 化运算提取特征，在解码过程中使用池化索引执行 非线性上采样, 减少内存占用并提升了速度. 为追 求模型轻量化, 实时语义分割算法 $\mathrm{ENet}^{[12]}$ 放弃最 后下采样阶段，感受野不足以覆盖比较大的对象， 导致算法分割精度较低，但是该算法具有分割速度 快的优点. 实时语义分割算法 $\mathrm{BiSeNet}^{[13]}$ 使用浅层 网络处理高分辨率图像，并提出一种快速下采样的 深层网络以平衡分类能力和感受野大小，此算法可 取得较高的分割速度和分割精度.
文献[14]指出浅层特征中存在不准确的语义 信息, 将深层和浅层特征直接叠加会产生大量噪 声，导致模型分割精度降低. 为解决此问题，本文 使用注意力机制为浅层特征分配像素级权重可抑 制不准确语义信息的输出. 除此之外, 本文认为输 人图像在神经网络中经下采样后得到的特征图中, 每个特征点在空间位置上与图像中的若干个像素 点组成的区域相对应, 而这些像素点所属的类别 可能不同，因此采用多标签分类损失函数显式地 监督训练网络, 使每个特征点可以具有多种类别 信息，提升特征语义信息的准确性，进而提升算法 分割精度.

\section{1 本文算法}

为使本文算法具有实时分割速度且具有较高 分割精度, 本文在当前最先进的语义分割算法 DeepLabv3 基础上轻量化特征提取网络, 即使用 ResNet34 $4^{[15]}$ 作为基础网络结构; 使用特征金字塔 结构为其增加解码结构, 使其具有实时分割速度; 将优化后的网络结构作为本文算法的基础网络, 同时增加注意力机制模块和多标签损失函数监督 训练, 进一步提升算法分割精度. 本节将详细介绍 这 3 点改进.

本文算法整体结构如图 1 所示, 其中 backbone 表示 ResNet34，/2 和 $\times 2$ 分别表示特征图下采样 2 倍和特征图上采样 2 倍, 虚线箭头表示在该阶段使 用多标签分类损失监督网络训练, “+”表示特征图 以相加的方式融合.

\section{1 神经网络结构}

在自然图像中的对象往往具有不同尺度和纵 横比, 如街景图像中天空、建筑、马路与路灯、广 告牌尺度差别较大, 具有不恰当感受野的神经网 络将无法给不同尺度目标均衡的关注. 比如, 具有 小感受野的神经网络将会更加关注小目标或者将 大目标分割成多个部分, 相反, 具有大感受野的神 经网络将忽视小目标. 因此, 获得多感受野网络对 于精细分割具有重要的意义，而 DeepLabv3 利用 图像的空间局部关联性和空洞卷积的采样特点对 图像进行卷积运算, 既可以获取多种感受视野信 息，又可以保留特征的空间信息; 但这导致大量参 数与高分辨率特征作点积运算, 降低分割速度. 而 本文在保留 DeepLabv3 多感受野性能的前提下对分 割速度进行优化. 本文算法整体结构如图 1 所示, 首先使用相对轻量级的 ResNet34 代替 DeepLabv3 


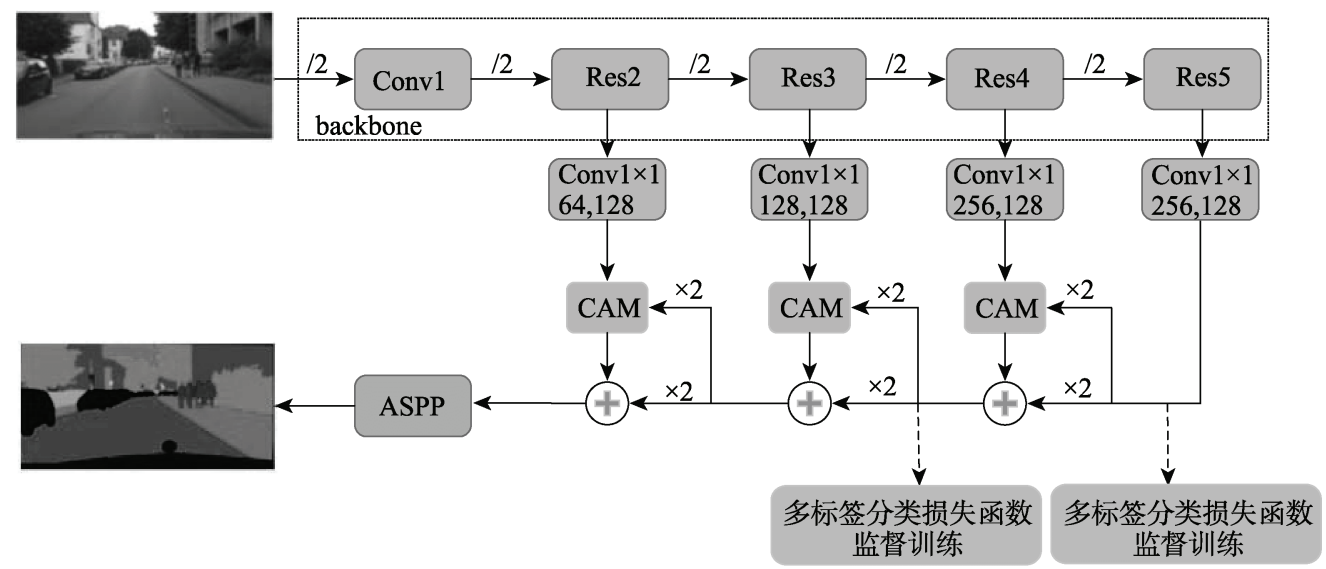

图 1 本文算法整体结构

中的 ResNet101 ${ }^{[15]}$ 作为特征编码器, 其次增加特征 金字塔网络 (feature pyramid networks, FPN) ${ }^{[16]}$ 作为 解码器逐层上采样恢复特征空间信息和语义信息, 最后压缩空洞金字塔池化网络中的参数数量. 其 中, ResNet34残差连接单元可避免梯度在反向传播 阶段消失；FPN 是一种融合不同层级特征图的方 式，解码阶段使用 FPN 重用浅层特征修复深层特 征图的空间细节信息，可以进一步增强特征图鲁 棒性. 如图 2 所示, ASPP 由不同空洞率 $r$ 的卷积并 联组成, 特征经过 FPN 处理之后具有较强的鲁棒 性, 因此只需要较少参数的 ASPP 模块即可实现多 尺度目标分割。

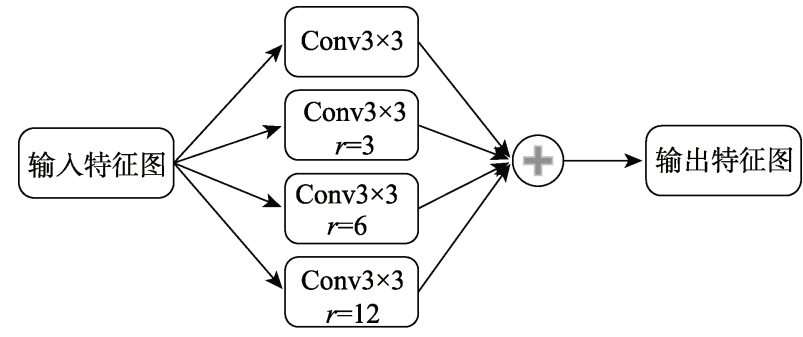

图 $2 \mathrm{ASPP}$

从网络组成方面来讲, 本文算法的网络主要 由卷积层、激活层、空洞卷积层和批标准化层(batch normalization, $\mathrm{BN})^{[17]}$ 共 4 种基础单元堆叠而成, 其 中卷积层负责提取图像特征，激活层负责提高网 络的非线性程度，空洞卷积层负责在保留特征空 间信息的前提下增大算法感受野、提升特征的鲁棒 性 ${ }^{[7]}$ ，而 $\mathrm{BN}$ 通过对网络不同层之间传递的数据进 行标准化以消除内部协变量移位现象 ${ }^{[17]}$, 进而提 高算法的收敛速度和精度.

\section{2 跨级注意力模块}

文献[14]指出，浅层特征中存在不准确的语义 信息，而 FPN 将深浅特征图直接相加，这种特征
融合方式将浅层特征中错误信息或冗余信息加到 深层特征，影响了算法分割精度. 鉴于此，在深浅 层特征融合的过程中，本文引人跨级注意力模块 (cross-level attention mechanism, CAM) 抑制错误信 息或冗余信息的输出. 注意力机制 ${ }^{[18]}$ 的作用机制 类似人类观察环境，往往只关注某些特别重要的 局部，获取不同局部的重要信息，抑制对当前识别 作用不大的特征, 增强有效特征的作用. 注意力特 征有助于增强模型的特征表达能力，综合不同信 息, 提高模型的理解能力 ${ }^{[19]}$.

本文提出的 CAM 如图 3 所示, 深层特征经过 $3 \times 3$ 卷积、 $B N$ 、激活处理后, 得到与编码器中浅层 特征图尺度相对应的可解释权重矩阵, 然后与浅 层特征图相乘，最后将加权后的浅层特征图与深 层特征图相加. 该模块以一种简单的方式使用深 层特征指导浅层特征加权, 为浅层特征图提供像 素级注意力, 使其关注更加具有信息量的特征点, 即在有限参数量下尽可能表达重要的信息. 该模块 能够更好地平衡修剪模型架构与增强模型表达力.

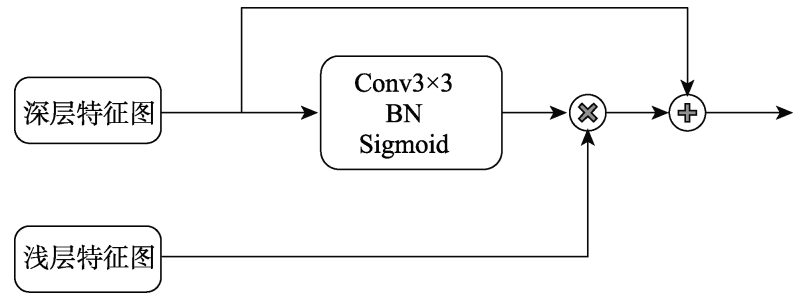

图 $3 \mathrm{CAM}$

\section{3 联合多标签分类监督训练}

不同于传统的单标签学习任务中每个样本只 与一种类别信息有关, 多标签学习 ${ }^{[20]}$ 需要输出多个 标签信息, 其中每个实例可以与一组标签相关联. 假设 $X=\mathbb{R}^{n}$ 表示 $n$ 维实例空间, $Y=\left\{y_{1}, y_{2}, \cdots, y_{q}\right\}$ 表示标签空间，该标签空间有 $q$ 种可能的标签类 
别. 多标签学习的任务是从多标签训练数据集 $D=$ $\left\{\left(x_{i}, Y_{i}\right) \mid 1 \leqslant i \leqslant m\right\}$ 中学习一个函数 $f: X \rightarrow 2^{Y}$, 对 于任意一个测试实例 $x \in X$, 多标签分类器 $f(\cdot)$ 预 测 $x$ 的标签集合 $f(x) \in Y$. 如图 4 所示，在单标签 分类任务中，可能将图 4 中箭头所指位置的标签分 配为马或者人，但是在多标签分类任务中，将会同 时分配这 2 种类别, 并以此作为神经网络的标签, 监督网络同时学习这 2 种类别的特征。

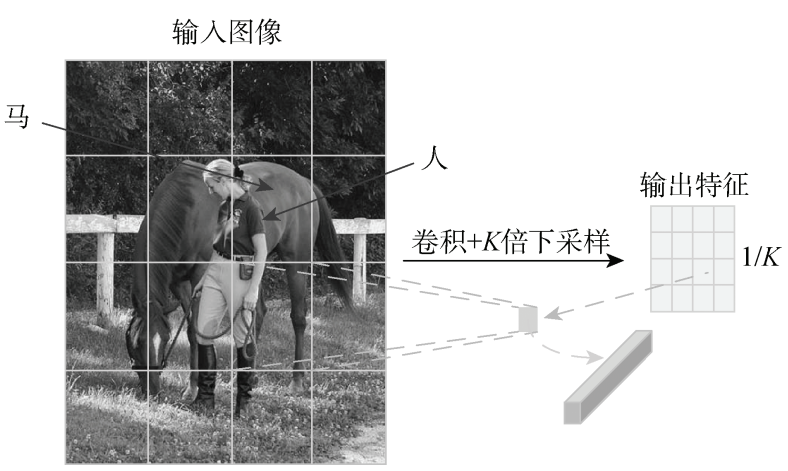

图 4 多标签分类图示

在当前语义分割技术中，使用线性插值对特 征进行上采样恢复语义信息类似于多标签分类任 务中提取特征的过程. 如图 4 所示, 在语义分割编 码阶段, 输人图像经卷积和下采样运算后输出原 图像 $1 / K$ 大小的特征图, 其中, $K$ 为下采样倍数. 图 4 中本文根据下采样倍数对图像按照空间位置划 分多个网格，根据卷积神经网络输出特征与原图 的映射关系可知，特征图中的每个特征点与每个 网格一一对应，与网格中的像素点为一对多的关 系. 通过图 4 可以发现, 在一部分网格中只存在背 景类别的像素, 另一部分网格中存在马、人、背景 共 3 种类别像素, 因此, 在某些网格内部存在多种 类别目标的边界交汇. 在解码器上采样阶段使用 线性插值对特征点采样提高特征图分辨率，由于 线性插值是基于空间不变模型的方法，无法捕捉 边缘快速变化的信息, 会产生边缘模糊效果 ${ }^{[21]}$.

因此，为进一步提高解码阶段上采样特征的 准确性，本文在特征图分辨率为原图 $1 / 32$ 和 $1 / 16$ 大小的特征图上进行上采样时, 引人多标签分类 损失函数显式地监督网络训练. 这样可以使特征 点包含的语义信息与图像中对应网格区域中的像 素类别信息一致，进而可以在恢复特征图分辨率 的同时保证类别信息准确性，并且不会降低算法 的分割速度. 本文使用多标签分类损失函数和交 叉熵损失函数共同监督网络学习，修正目标边界 信息. 损失函数描述为

$$
\begin{aligned}
L= & \lambda_{1} L_{\mathrm{CE}}(y, \hat{y})+\lambda_{2} L_{\mathrm{BCE}}\left(s_{16}, \hat{s}_{16}\right)+ \\
& \lambda_{3} L_{\mathrm{BCE}}\left(s_{32}, \hat{s}_{32}\right) .
\end{aligned}
$$

其中， $L_{\mathrm{CE}}$ 表示交叉熵损失函数; $L_{\mathrm{BCE}}$ 表示多标签 损失函数，本文中多标签损失函数使用二进制交 叉熵损失函数； $y, s_{16}, s_{32} \in \mathbb{R}^{H \times W}$ 表示真实标签; $s_{16}$ 和 $s_{32}$ 表示标签分辨率大小分别为原标签分辨 率大小的 $1 / 16$ 和 $1 / 32 ; \hat{y}, \hat{s}_{16}, \hat{s}_{32} \in \mathbb{R}^{H \times W}$ 表示对应 预测值; $\lambda_{1}, \lambda_{2}, \lambda_{3}$ 表示控制 3 个损失函数权重的 3 个超参数

\section{2 实验与分析}

\section{1 评价指标}

在本文实验中使用平均交并比(mean intersection-over-union, mIoU), 处理每幅图像所用时间 $t(\mathrm{~ms})$ 和图像处理速度 $v($ 帧/s) 作为算法性能评价 指标.

$\mathrm{mIoU}$ 为语义分割的标准度量, 计算 2 个集合 的交集与并集之比，在每个像素类别内计算交并 比(intersection-over-union, IoU), 然后计算平均值. 使用处理每幅图像所用的时间 $t(\mathrm{~ms})$ 和图像处理速 度 $v($ 帧 $/ \mathrm{s})$ 来衡量算法的速度, $\mathrm{mIoU}$ 和 $v$ 计算公式 分别为

$$
\begin{aligned}
& \mathrm{mIoU}=\frac{1}{k+1} \sum_{i=0}^{k} \frac{p_{i i}}{\sum_{j=0}^{k} p_{i j}+\sum_{j=0}^{k} p_{j i}-p_{i i}}, \\
& v=\frac{N}{\sum_{i}^{N} t_{i}} .
\end{aligned}
$$

其中, $p_{i i}$ 表示分割正确的数量; $p_{i j}$ 表示本属于 $i$ 类但预测为 $j$ 类的像素数量; $p_{j i}$ 表示本属于 $j$ 类 被预测为 $i$ 类的像素数量; $N$ 表示图像数量; $t$ 表 示处理每幅图像所用的时间.

\section{2 实验数据与实验环境}

Cityscapes 数据集 ${ }^{[22]}$ 包含来自不同城市、不同 季节拍摄的 5000 幅精确标注和 20000 幅粗略标注 的街景图像, 每幅图像分辨率为 1024 像素 $\times 2048$ 像 素. 数据集共 19 个街景类别. 在本文实验中，仅使 用精确标注的 5000 幅图像, 其中 3475 幅用于训练 模型, 1525 幅用于测试, 测试数据没有提供真实标 签, 需要提交其官方服务器测评.

CamVid 数据集 ${ }^{[23]}$ 包含从视频序列中提取的 701 幅分辨率为 760 像素 $\times 960$ 像素的图像, 其中 367 幅用于训练, 101 幅用于验证, 233 幅用于测试, 
在本文实验中共测试 11 个语义类别.

本文所有实验的仿真实验环境为 Ubuntu 18.04, Python3.7.4, Pytorch1.1.0, 显卡为 NVIDIA Titan RTX 和 GTX1060. 模型编码器基础网络是在 ImageNet 数据集 ${ }^{[24]}$ 上预训练的 ResNet34. 初始学 习速率设置为 0.005 , 学习速率调整策略使用多项 式衰减策略, 权重衰减使用 $L_{2}$ 正则化, 衰减系数 设置为 0.0005 , 动量设置为 0.9 .

\section{3 算法性能分析与比较}

首先，本文基于 Cityscapes 和 CamVid 数据集 的分割结果在速度和精度 2 个方面进行模块有效 性评估, 之后与模型 FCN-8s, DeepLabv2, ENet, SegNet, ICNet ${ }^{[25]}$ 和 BiSeNet 对比, 最后通过可视化 结果进一步分析算法的分割性能.

\subsection{1 模块有效性评估}

为评估本文提出的 CAM 的效果, 首先对轻量 化后的 DeepLabv3 模型进行评估, 记为 Baseline, 然后对应用了本文 CAM 模型进行评估, 记为 Baseline + CAM. 表 1 所示为在显卡为 NVIDIA Titan RTX 的实验环境下的消融实验结果, 在 Cityscapes 和 CamVid 数据集上的实验结果显示, 引人该 $\mathrm{CAM}$ 后 $\mathrm{mIoU}$ 分别提高了 $1.2 \%$ 和 $1.4 \%$, 处 理每幅图像的运算时间分别增加 $1.7 \mathrm{~ms}$ 和 $1.4 \mathrm{~ms}$, 证明该注意力模块可以在消耗很少运算时间的前 提下提高模型分割精度, 并且说明深层特征图可 以有效地指导浅层特征图保留有效信息，防止传 人过多干扰信息.

表 1 各模块有效性评估对比表

\begin{tabular}{lccccc}
\hline \multirow{2}{*}{ 算法 } & \multicolumn{2}{c}{ Cityscapes $(768 \times 1536)$} & & \multicolumn{2}{c}{ CamVid(720×960) } \\
\cline { 2 - 3 } \cline { 6 - 6 } \cline { 5 - 6 } & $\mathrm{mIoU} / \%$ & $t / \mathrm{ms}$ & & $\mathrm{mIoU} / \%$ & $t / \mathrm{ms}$ \\
\hline Baseline & 65.3 & 21.9 & & 71.5 & 9.8 \\
Baseline+CAM & 66.5 & 23.6 & & 72.9 & 11.2 \\
Baseline $+\mathrm{CAM}+L_{\mathrm{ML}}$ & 68.1 & 23.6 & & 74.1 & 11.2 \\
\hline
\end{tabular}

为评估本文提出的多标签分类损失函数辅助 监督算法 (记为 Baseline $+\mathrm{CAM}+L_{\mathrm{ML}}$ ) 的有效性, 将 仅使用交叉熵损失函数的算法与在解码器阶段使 用多标签分类损失函数的算法对比. 其中, 关于多 标签损失函数的超参数设定, 本文通过经验方式 人为确定几组不同超参数值对比网络性能, 从中 选取一组较优的超参数作为本文多标签损失函数 的超参数, 最终超参数设定为 $\lambda_{1}=1, \lambda_{2}=0.3$, $\lambda_{3}=0.7$. 由表 1 可以看出, 相对于仅使用交叉熵 损失函数的算法，使用多标签分类函数监督网络 训练的精度在 Cityscapes 和 CamVid 数据集上分别
提高了 $1.6 \%$ 和 $1.2 \%$, 并且对网络运行速度没有影 响, 可以说明本文采用多标签分类损失函数监督 网络解码训练的有效性. 此外, 通过改变网络监督 方式提升网络性能, 对于图像实时语义分割是一 种非常有效的方式，既能提高分割精度又不会影 响分割速度.

\subsection{2 算法整体分析与比较}

为验证本文算法的有效性, 实验将本文算法 与 DeepLabv2, FCN-8s, SegNet, ENet 在 Cityscapes 和 CamVid 数据集上进行分割精度和速度的对比, 统一采用 $\mathrm{mIoU}$ 衡量语义分割精度, 训练参数设置 见第 2.2 节.

从算法分割精度和处理速度上分析，在 Cityscapes 数据集上使用分辨率为 768 像素 $\times 1536$ 像素, 512 像素 $\times 1024$ 像素进行训练、测试, 在 CamVid 数据集上分别使用 720 像素 $\times 960$ 像素, 384 像素 $\times 480$ 像素图像进行训练和测试. 表 2 是在显 卡为 NVIDIA Titan RTX 的实验环境下的部分实验 结果. 其中, 本文算法在 2 个数据集上的处理速度 分别为 42 帧/s 和 89 帧/s, 虽然分割速度略慢于 ENet, 但是在 2 个数据集上的 $\mathrm{mIoU}$ 比 ENet 分别 提高了 $9.8 \%$ 和 $5.8 \%$. 与 SegNet 相比, 在速度更快 的前提下, 在 2 个数据集上的 $\mathrm{mIoU}$ 提高了 $11.1 \%$ 和 $8.9 \%$. 可见，本文算法在速度与精度上与现有 的实时分割算法相比有较好的表现. 与 DeepLabv2 和 FCN-8s 非实时语义分割模型相比, 本文算法的 分割精度也具有较大优势. 综合分析, 本文算法可 以在分割速度与分割精度之间取得较好的平衡, 可以实现精确高效的分割.

表 2 不同算法在 2 个数据集上的性能对比

\begin{tabular}{|c|c|c|c|c|c|c|}
\hline \multirow{2}{*}{ 算法 } & \multicolumn{3}{|c|}{ Cityscapes $(768 \times 1536)$} & \multicolumn{3}{|c|}{ CamVid( $(720 \times 960)$} \\
\hline & $\mathrm{mIoU} / \%$ & $t / \mathrm{ms}$ & $v /\left(\right.$ 帧 $\left.\cdot \mathrm{s}^{-1}\right)$ & $\mathrm{mIoU} / \%$ & $t / \mathrm{ms}$ & $v /\left(\right.$ 帧 $\left.\cdot \mathrm{s}^{-1}\right)$ \\
\hline DeepLabv2 ${ }^{[8]}$ & 63.1 & 4000.2 & $<1$ & 71.3 & 830.0 & 1 \\
\hline $\mathrm{FCN}-8 \mathrm{~s}^{[4]}$ & 60.4 & 250.0 & 4 & 66.9 & 76.9 & 13 \\
\hline $\operatorname{SegNet}^{[11]}$ & 57.0 & 31.3 & 32 & 65.2 & 13.5 & 74 \\
\hline $\mathrm{ENet}^{[12]}$ & 58.3 & 11.9 & 83 & 68.3 & 7.3 & 136 \\
\hline 本文-ResNet34 & 68.1 & 23.6 & 42 & 74.1 & 11.2 & 89 \\
\hline
\end{tabular}

图 5 所示为本文算法在 2 个数据集上训练的损 失函数曲线, 其中非联合多标签分类的交叉熵损 失仅使用交叉摘损失. 可以看出, 使用本文提出的 多标签分类损失函数作为损失函数辅助训练的算 法在训练过程中损失值平稳下降, 相较于仅使用 交叉熵损失函数有更好的表现，进而可有效地监 督算法在低分辨率特征图上进行多标签分类. 


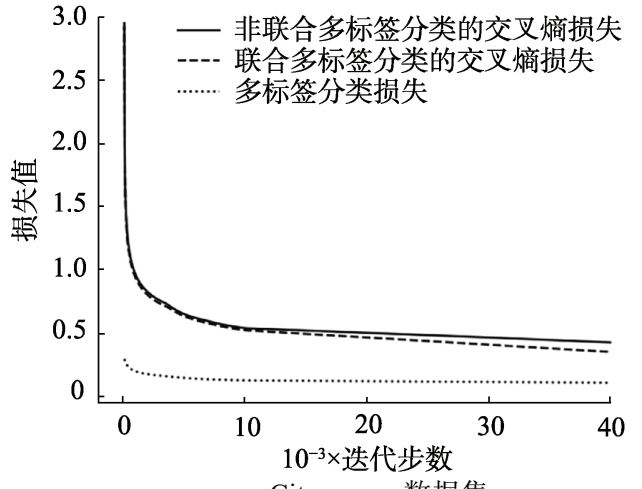

a. Cityscapes 数据集

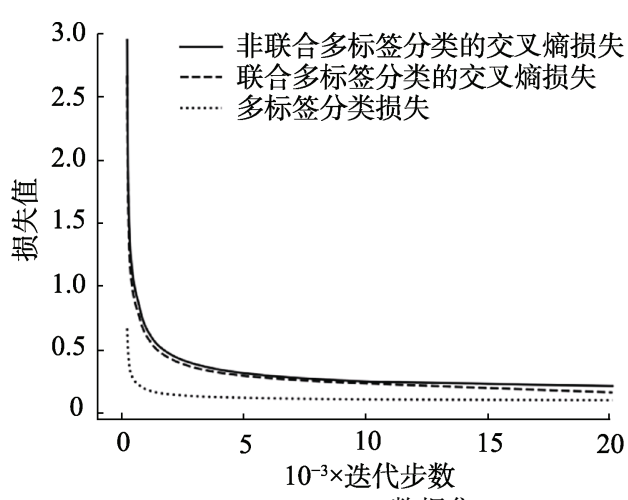

b. CamVid 数据集

图 5 不同算法在 2 个数据集上的损失对比曲线

表 3 和表 4 为 6 GB 显存 GTX1060 显卡实验 环境下的实验性能对比, 训练过程中无任何数据 扩充, 图 6 图 8 所示为相应分割效果展示图. 表 3 和图 6 分别展示本文算法(本文-ResNet34)与 ICNet, BiSeNet 在 Cityscapes 数据集上的分割结果对比. 根据表 3 在 Cityscapes 和 CamVid 数据集上的分割 实验数据可知, 相比于 ICNet, 本文算法在分割精 度和分割速度上均有较大优势. 相比于 BiSeNet, 本文算法分割精度有所提高, 但是分割速度稍慢. 根据图 6 分割效果图可以看出, 从分割细节来讲, ICNet 丢失细节较多, BiSeNet 对细节分割好于 ICNet，而本文算法好于 BiSeNet，这一点可以通过 图 6 中的杆状目标分割效果看出; 另一方面, 这 3 种实时分割算法对图像的整体均能取得较好的分 割效果，但依据图 6 第 2 行和第 3 行的展示图可以 看出，本文算法和 BiSeNet 对马路的分割效果好于
ICNet. 而 Baseline-ResNet34 虽然对细节分割效果 好于 ICNet, 但对图像整体分割效果较差.

表 3 不同算法在 2 个数据集上性能对比

\begin{tabular}{|c|c|c|c|c|c|c|}
\hline \multirow{2}{*}{ 算法 } & \multicolumn{3}{|c|}{ Cityscapes $(512 \times 1024)$} & \multicolumn{3}{|c|}{ CamVid $(360 \times 480)$} \\
\hline & $\mathrm{mIoU} / \%$ & $t / \mathrm{ms}$ & $v /\left(\right.$ 帧 $\left.\cdot \mathrm{s}^{-1}\right)$ & $\mathrm{mIoU} / 0$ & $t / \mathrm{ms}$ & $v /\left(\right.$ 帧 $\left.\cdot \mathrm{s}^{-1}\right)$ \\
\hline $\mathrm{ICNet}^{[25]}$ & 65.3 & 60.0 & 16 & 63.4 & 40.0 & 25 \\
\hline $\mathrm{BiSeNet}^{[13]}$ & 66.2 & 20.6 & 51 & 65.1 & 14.9 & 67 \\
\hline 本文-ResNet34 & 66.8 & 23.0 & 43 & 65.3 & 17.0 & 59 \\
\hline
\end{tabular}

表 4 本文算法和 DeepLabv3 使用

MobileNetv2 性能对比

\begin{tabular}{|c|c|c|c|c|c|c|}
\hline \multirow{2}{*}{ 算法 } & \multicolumn{3}{|c|}{ Cityscapes $(512 \times 1024)$} & \multicolumn{3}{|c|}{ CamVid $(360 \times 480)$} \\
\hline & $\mathrm{mIoU} / \%$ & $t / \mathrm{ms}$ & $v /\left(\right.$ 帧 $\left.\cdot \mathrm{s}^{-1}\right)$ & $\mathrm{mIoU} / \%$ & $t / \mathrm{ms}$ & (帧· $\mathrm{s}^{-1}$ ) \\
\hline $\begin{array}{l}\text { Deeplabv3- } \\
\text { MobileNetv2 }\end{array}$ & 64.3 & 30.2 & 31 & 62.3 & 22.2 & 45 \\
\hline 本文-MobileNetv2 & 65.4 & 20.0 & 50 & 63.9 & 15.9 & 63 \\
\hline
\end{tabular}
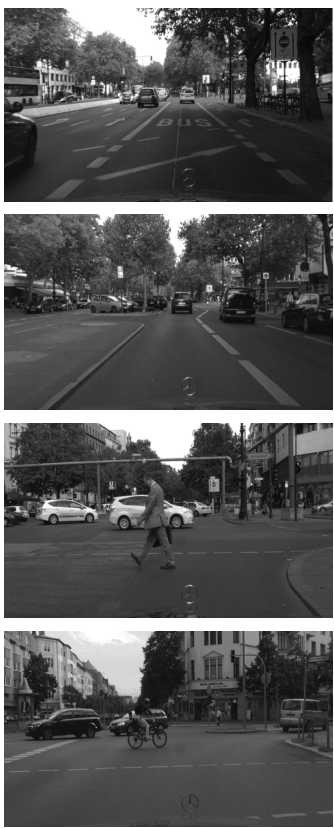

a. 输人图像
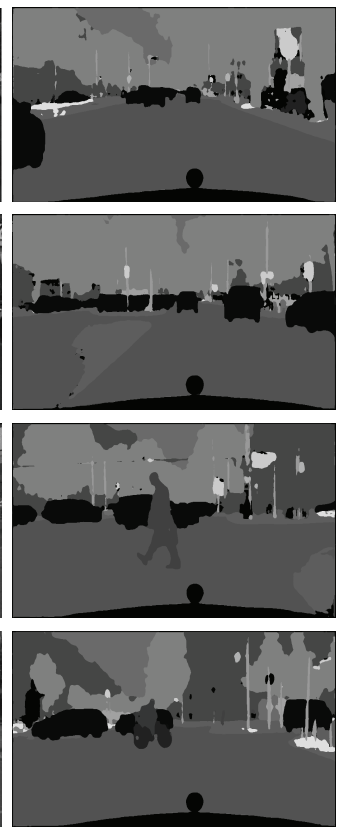

b. Baseline-ResNet34
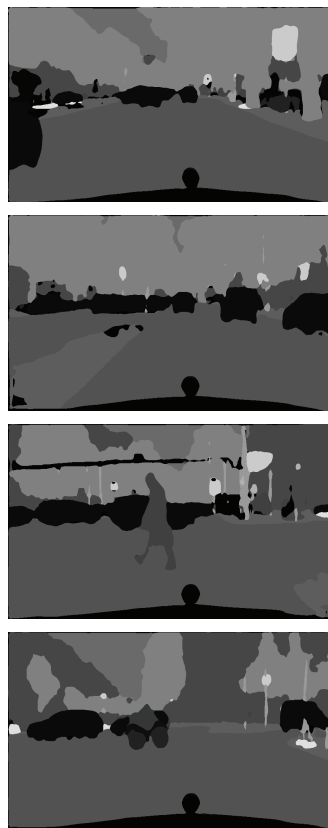

c. $\mathrm{ICNet}^{[25]}$
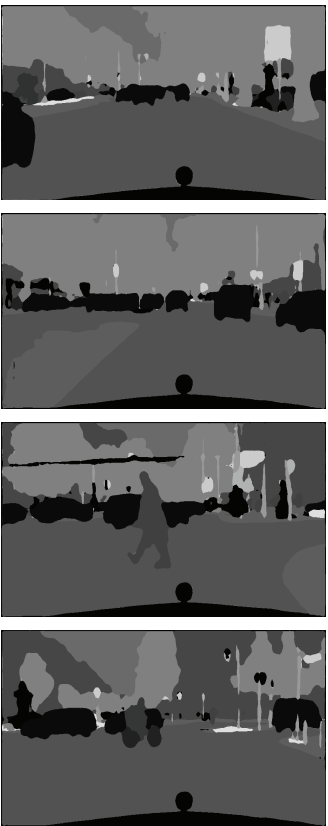

d. $\mathrm{BiSeNet}^{[13]}$
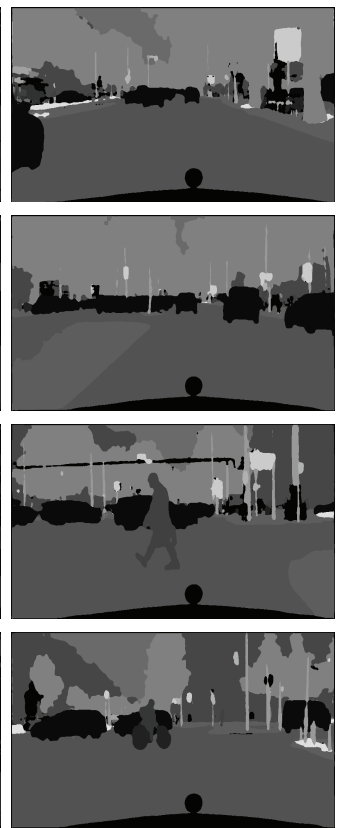

e. 本文-ResNet34

图 6 实时语义分割算法结果对比 
本文算法与 DeepLabv3 使用 MobileNetv2 ${ }^{[26]}$ 作为特征提取网络的分割效果对比如图 7 所示, 其 对应的实验数据如表 4 所示. 从图 7 可以看出, 本 文算法在分割细节方面稍好于 DeepLabv3，但 DeepLabv3 的分割效果表现出该模型丢失了图像 中更多的小目标和细节现象; 从图像整体分割效 果来看, 这 2 种模型均能取得较好的分割效果.

再结合图 6 和图 7 分析 ResNet34 和 MobileNetv2 作为特征提取网络对本文算法分割性能的影响.
可以看出, 使用 ResNet34 作为特征提取网络的模 型在图像细节分割效果上要好于使用 MobileNetv2 的模型, 在图像整体分割效果上二者分割效果大 体相似. 而结合表 3 和表 4 数据可以看出, 本文ResNet34 与本文-MobileNetv2 模型分割速度相近. 本文分析这与测试 Batch 大小有关, 本文实验测试 Batch 为 1 , 当 Batch 增大, 本文-MobileNetv2 模型 占用显存少的优势更明显, 可被并行处理的图像 数量增多, 其分割速度会有显著提升.
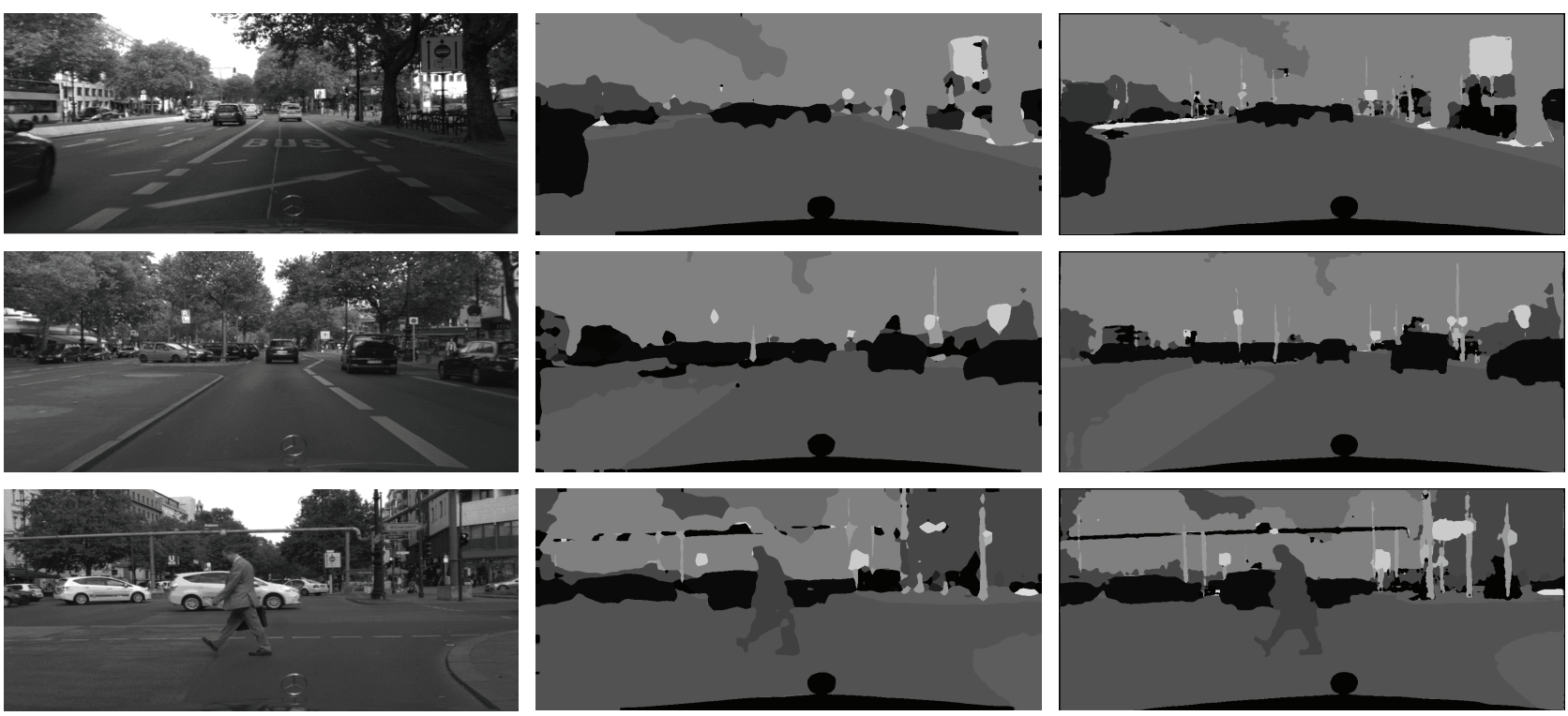

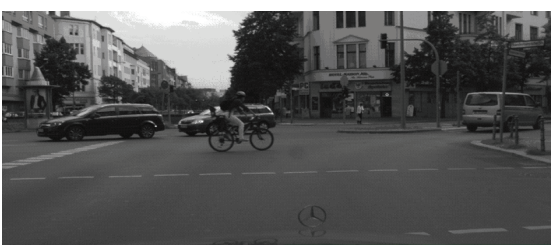

a. 输人图像

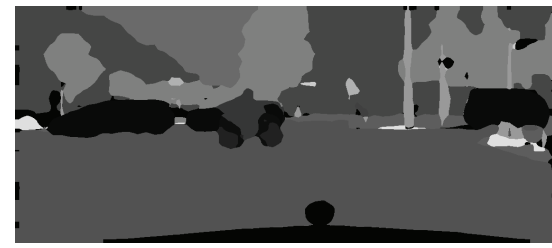

b. Deeplabv3-MobileNetv2

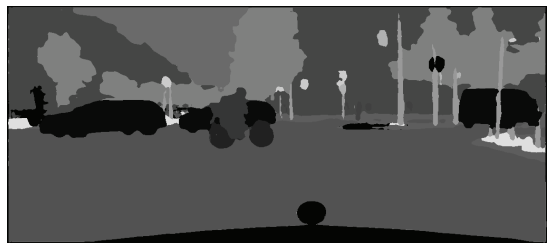

c. 本文-MobileNetv2

图 7 Deeplabv3 与本文算法在 Cityscapes 数据集上的对比

接下来通过图 8 分析本文算法性能. 图 8c 所 示为 Baseline, 其提取特征网络为 ResNet34, 该模 型不含有 CAM 和多标签分类; 图 $8 \mathrm{~d}$ 所示为使用 MobileNetv2 作为提取特征网络的 DeepLabv3 模 型; 图 $8 \mathrm{e}$ 和图 $8 \mathrm{f}$ 分别为使用 MobileNetv2 和 ResNet34 作为提取特征网络并与 CAM 和多标签分 类相结合的模型，即本文最终模型. 对于 CamVid 数据集, 其图像中细节部分较多, 实验中的图像分 辨率只有 360 像素 $\times 480$ 像素, 此分辨率较低, 因此 在该数据集进行实验更加考察模型分割图像细节 的能力. 从图 8 可以看出, Baseline 对细节丢失最 多, 而本文算法和 DeepLabv3 对细节的分割要好 于 Baseline，可见本文算法的有效性.

\section{3 结 语}

本文提出了一种基于注意力机制和多标签分 类的实时图像语义分割网络, 首先优化 DeepLabv3 神经网络架构至满足实时分割的要求，在此基础 上设计了跨级特征注意力模块和多标签分类损失 函数. CAM 利用蕴含丰富语义信息的深层特征对 浅层特征进行像素级加权, 实现更精确的空间信 息选取, 同时, 使用多标签分类损失函数辅助监督 网络学习，在恢复特征图分辨率时有效提高类别 信息准确性，二者共同作用使得类别边界像素分 割更精细. 最后, 在 Cityscapes 数据集和 CamVid 数据集上进行了一系列对比实验, 实验结果表明, 本文算法能够更加准确地处理复杂场景图像中图 

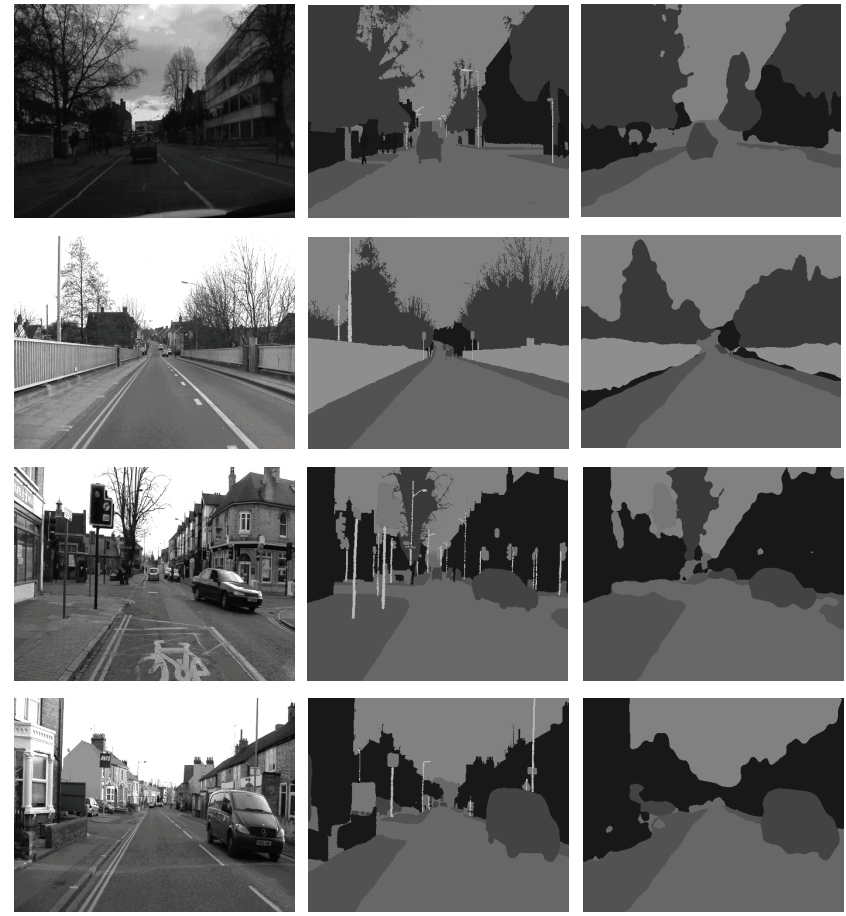

a. 输人图像

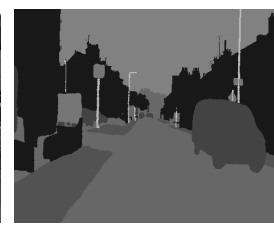

b. 真实标签

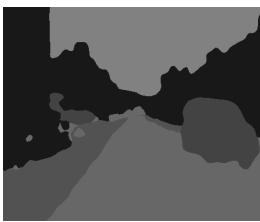

c. Baseline
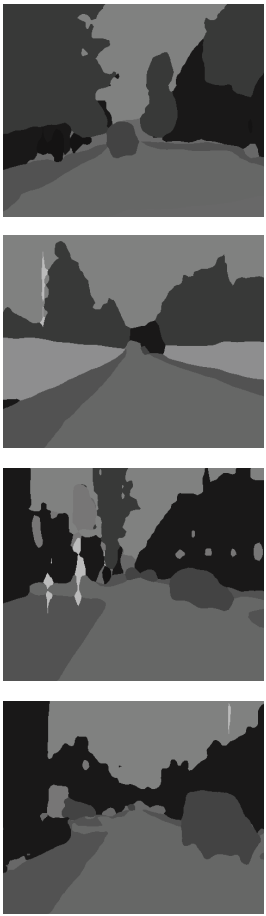

d. DeepLabv3
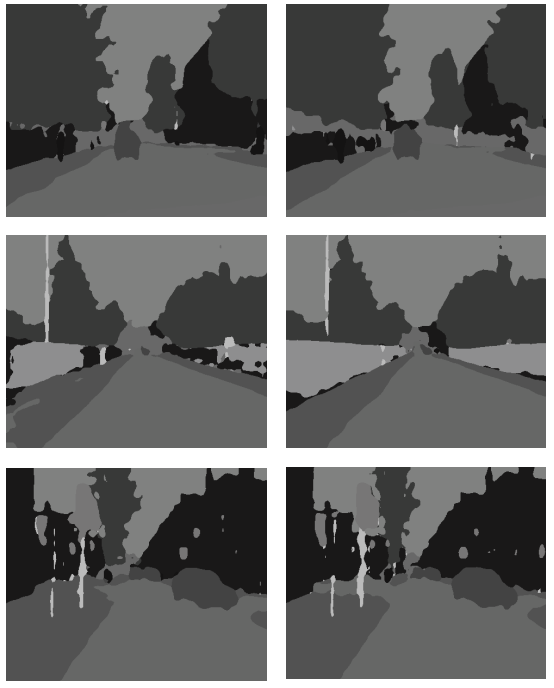

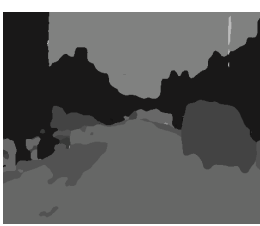

e. 本文-MobileNetv2

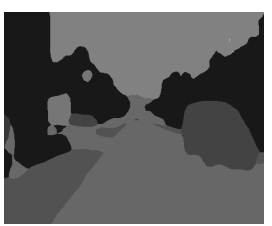

f. 本文-ResNet34

图 8 DeepLabv3 与本文算法在 CamVid 数据集上的对比

像分割问题，显著改善类别边缘区域分割效果，同 时证明本文算法是一种分割精度高、分割速度快的 图像实时语义分割算法.

\section{参考文献(References):}

[1] Csurka G, Perronnin F. An efficient approach to semantic segmentation[J]. International Journal of Computer Vision, 2011, 95(2): 198-212

[2] He Y H, Wang H, Zhang B. Color-based road detection in urban traffic scenes[J]. IEEE Transactions on Intelligent Transportation Systems, 2004, 5(4): 309-318

[3] An Zhe, Xu Xiping, Yang Jinhua, et al. Design of augmented reality head-up display system based on image semantic segmentation[J]. Acta Optica Sinica, 2018, 38(7): 77-83(in Chinese)

(安喆, 徐熙平, 杨进华, 等. 结合图像语义分割的增强现实 型平视显示系统设计与研究 [J]. 光学学报, 2018, 38(7): 77-83)

[4] Long J, Shelhamer E, Darrell T. Fully convolutional networks for semantic segmentation[C] //Proceedings of the IEEE Conference on Computer Vision and Pattern Recognition. Los Alamitos: IEEE Computer Society Press, 2015: 3431-3440

[5] Lin G S, Milan A, Shen C H, et al. Refinenet: multi-path refinement networks for high-resolution semantic segmentation $[\mathrm{C}] / /$ Proceedings of the IEEE Conference on Computer Vision and Pattern Recognition. Los Alamitos: IEEE Computer Society Press, 2017: 5168-5177

[6] Ronneberger O, Fischer P, Brox T. U-Net: convolutional networks for biomedical image segmentation[C] //Proceedings of
Medical Image Computing and Computer Assisted Intervention. Heidelberg: Springer, 2015: 234-241

[7] Chen L C, Papandreou G, Kokkinos I, et al. Semantic image segmentation with deep convolutional nets and fully connected crfs[OL]. [2020-02-16]. https://arxiv.org/abs/1412.7062, 2014

[8] Chen L C, Papandreou G, Kokkinos I, et al. DeepLab: semantic image segmentation with deep convolutional nets, atrous convolution, and fully connected CRFs[J]. IEEE Transactions on Pattern Analysis and Machine Intelligence, 2018, 40(4): 834-848

[9] Chen L C, Papandreou G, Schroff F, et al. Rethinking atrous convolution for semantic image segmentation[OL]. [2020-02-16]. https://arxiv.org/abs/1706.05587

[10] Yue Shiyi. Image semantic segmentation based on hierarchical context information[J]. Laser \& Optoelectronics Progress, 2019, 56(24): 107-115 (in Chinese)

(岳师怡. 基于多层级上下文信息的图像语义分割 [J]. 激光 与光电子学进展, 2019, 56(24): 107-115)

[11] Badrinarayanan V, Kendall A, Cipolla R. SegNet: a deep convolutional encoder-decoder architecture for image segmentation[J]. IEEE Transactions on Pattern Analysis and Machine Intelligence, 2017, 39(12): 2481-2495

[12] Paszke A, Chaurasia A, Kim S, et al. ENet: a deep neural network architecture for real-time semantic segmentation[OL]. [2020-02-16]. https://arxiv.org/abs/1606.02147

[13] Yu C Q, Wang J B, Peng C, et al. BiSeNet: bilateral segmentation network for real-time semantic segmentation[C] //Proceedings of the European Conference on Computer Vision. Heidelberg: Springer, 2018: 334-349

[14] Ghiasi G, Fowlkes C C. Laplacian pyramid reconstruction and refinement for semantic segmentation[C] //Proceedings of the European Conference on Computer Vision. Heidelberg: Springer, 2016: 519-534 
[15] He K M, Zhang X Y, Ren S Q, et al. Deep residual learning for image recognition[C] //Proceedings of the IEEE Conference on Computer Vision and Pattern Recognition. Los Alamitos: IEEE Computer Society Press, 2016: 770-778

[16] Lin T, Dollar P, Girshick R, et al. Feature pyramid networks for object detection[C] //Proceedings of the IEEE Conference on Computer Vision and Pattern Recognition. Los Alamitos: IEEE Computer Society Press, 2017: 936-944

[17] Ioffe S, Szegedy C. Batch normalization: accelerating deep network training by reducing internal covariate shift[OL]. [2020-02-16]. https://arxiv.org/abs/1502.03167

[18] Vaswani A, Shazeer N, Parmar N, et al. Attention is all you need[C] //Proceedings of the Neural Information Processing Systems. New York: Curran Associates, 2017: 5998-6008

[19] Feng Xingjie, Zhang Le, Zeng Yunze. Question similarity calculation model based on multi-attention $\mathrm{CNN}[\mathrm{J}]$. Computer Engineering, 2019, 45(9): 284-290(in Chinese)

(冯兴杰, 张乐, 曾云泽. 基于多注意力 $\mathrm{CNN}$ 的问题相似度 计算模型[J]. 计算机工程, 2019, 45(9): 284-290)

[20] Jiang Siyu, Zhong Xiaoling, Qiu Shaojian, et al. A multi-label learning model based on label correlation and imbalance[J]. Journal of Harbin Institute of Technology, 2019, 51(1): 142-149(in Chinese)

(姜思羽, 钟晓玲, 邱少健, 等. 结合标签相关性和不均衡性
的多标签学习模型 $[\mathrm{J}]$. 哈尔滨工业大学学报, 2019, 51(1): 142-149)

[21] Li X, Orchard M T. New edge-directed interpolation[J]. IEEE Transactions on Image Processing, 2001, 10(10): 1521-1527

[22] Cordts M, Omran M, Ramos S, et al. The Cityscapes dataset for semantic urban scene understanding[C] //Proceedings of the IEEE Conference on Computer Vision and Pattern Recognition. Los Alamitos: IEEE Computer Society Press, 2016: 3213-3223

[23] Brostow G J, Fauqueur J, Cipolla R. Semantic object classes in video: a high-definition ground truth database[J]. Pattern Recognition Letters, 2009, 30(2): 88-97

[24] Deng J, Dong W, Socher R, et al. ImageNet: a large-scale hierarchical image database[C] //Proceedings of the IEEE Conference on Computer Vision and Pattern Recognition. Los Alamitos: IEEE Computer Society Press, 2009: 248-255

[25] Zhao H S, Qi X J, Shen X Y, et al. ICNet for real-time semantic segmentation on high-resolution images[C] //Proceedings of the European Conference on Computer Vision. Heidelberg: Springer, 2018: 418-434

[26] Sandler M, Howard A, Zhu M L, et al. MobileNetv2: inverted residuals and linear bottlenecks[C] //Proceedings of the IEEE Conference on Computer Vision and Pattern Recognition. Los Alamitos: IEEE Computer Society Press, 2018: 4510-4520 